\title{
Anti-biofilm activity of pseudoalteromonas haloplanktis tac I 25 against staphylococcus epidermidis biofilm: Evidence of a signal molecule involvement?
}

International Journal of Immunopathology and Pharmacology 2015, Vol. 28(I) 104-113 (c) The Author(s) 2015 Reprints and permissions: sagepub.co.uk/journalsPermissions.nav DOI: $10.1177 / 039463201557275$ | iji.sagepub.com

@SAGE

\author{
E Parrilli,' R Papa, ${ }^{2} S$ Carillo,' M Tilotta, ${ }^{2}$ A Casillo,' F Sannino, 1,3 \\ A Cellini, ${ }^{2}$ M Artini, ${ }^{2}$ L Selan, ${ }^{2}$ MM Corsaro' and ML Tutino'
}

\begin{abstract}
Staphylococcus epidermidis is recognized as cause of biofilm-associated infections and interest in the development of new approaches for $S$. epidermidis biofilm treatment has increased. In a previous paper we reported that the supernatant of Antarctic bacterium Pseudoalteromonas haloplanktis TACI25 presents an anti-biofilm activity against S. epidermidis and preliminary physico-chemical characterization of the supernatant suggested that this activity is due to a polysaccharide. In this work we further investigated the chemical nature of the anti-biofilm $P$. haloplanktis TACI25 molecule. The production of the molecule was evaluated in different conditions, and reported data demonstrated that it is produced in all $P$. haloplanktis TACI 25 biofilm growth stages, also in minimal medium and at different temperatures. By using a surface coating assay, the surfactant nature of the anti-biofilm compound was excluded. Moreover, a purification procedure was set up and the analysis of an enriched fraction demonstrated that the anti-biofilm activity is not due to a polysaccharide molecule but that it is due to small hydrophobic molecules that likely work as signal. The enriched fraction was also used to evaluate the effect on S. epidermidis biofilm formation in dynamic condition by BioFlux system.
\end{abstract}

\section{Keywords \\ antibiofilm, Bioflux, dynamic biofilm assay, P. haloplanktis TACI25, S. epidermidis biofilm}

Received I6 September 2014; accepted I5 December 2014

Staphylococcus epidermidis ( $S$. epidermidis) is now being recognized as an important opportunistic pathogen that can cause significant problems when breaching the epithelial barrier, especially during biofilm-associated infection of indwelling medical devices. ${ }^{1,2}$ Most diseases caused by S. epidermidis are of a chronic character and occur as device-related infections (such as intravascular catheter or prosthetic joint infections) and/or their complications. ${ }^{2}$ The ability of this bacterium to adhere on both eukaryotic cells and abiotic surfaces and to form biofilm is an essential virulence factor. This capacity contributes to making S. epidermidis infections chronic and particularly difficult to eradicate.

Biofilms are sticky, surface-attached agglomerations of bacteria that are embedded in an extracellular matrix and provide protection from antibiotics and mechanisms of host defense. ${ }^{3}$ Considering the impact of $S$. epidermidis bacterial biofilms on human health, coagulase-negative staphylococci bloodstream infections originating from intravascular catheter infections are estimated to reach 250,000 cases per year in the USA with a mortality rate of $1-25 \% .{ }^{4}$ Interest in the development of innovative approaches for the

\footnotetext{
'Department of Chemical Sciences, Federico II University, Naples, Italy 2Department of Public Health and Infectious Diseases, Sapienza University, Rome, Italy

3 Institute of Protein Biochemistry, CNR, Naples, Italy

Corresponding author:

Ermenegilda Parrilli, Department of Chemistry, Federico II University, Complesso Universitario Monte Sant'Angelo, Via Cinthia 4, 80I26

Naples, Italy.

Email: erparril@unina.it
} 
prevention and treatment of staphylococcal adhesion and biofilm formation capabilities has increased. A viable approach should target staphylococcal adhesive properties without affecting bacterial vitality in order to avoid the rapid appearance of escape mutants.

From another point of view, the biofilm could be considered as a source of novel drugs. Indeed, the specific environmental conditions prevailing within biofilms may induce profound genetic and metabolic rewiring of the biofilm-dwelling bacteria and therefore can allow the production of metabolites different from those obtained in planktonic condition. Furthermore, many bacterial biofilms secrete molecules such as quorum sensing signals, ${ }^{5}$ surfactants, ${ }^{6}$ enzymes, ${ }^{7}$ and polysaccharides $^{8,9}$ that act by regulating biofilm architecture or mediating the release of cells from biofilms during the dispersal stage of the biofilm life cycle. ${ }^{7}$ Also, the production of extracellular molecules that degrade adhesive components in the biofilm matrix is a basic mechanism used in the biological competition between phylogenetically different bacteria. ${ }^{10-12}$

Marine bacteria belonging to the genus Pseudoalteromonas produce compounds of biotechnological interest, including anti-biofilm molecules. ${ }^{13}$ Marine bacteria from Antarctica represent an untapped reservoir of biodiversity; indeed, Antarctic microorganisms can synthesize a broad range of potentially valuable bioactive compounds. ${ }^{14-16}$

The present authors previously reported that $P$. haloplanktis TAC125 strain holds an anti-biofilm activity; ${ }^{15}$ this bacterium had been isolated from Antarctic sea water near Terre Adelie. ${ }^{17}$ The antibiofilm activity of cell-free supernatant of $P$. haloplanktis grown in static and in planktonic condition was tested on different staphylococci. The results obtained demonstrated that only supernatant of $P$. haloplanktis grown in static condition inhibits biofilm of $S$. epidermidis but it was not effective on $S$. aureus biofilm. This anti-biofilm activity impairs biofilm development and disaggregates the mature biofilm of $S$. epidermidis without affecting bacterial viability, showing that its action is specifically directed against biofilm. A preliminary chemical characterization of the biofilm-inhibiting compound suggests that the biologically active component could be a polysaccharide. ${ }^{15}$ The aim of the present work is to further investigate the chemical nature of $P$. haloplanktis TAC125 anti-biofilm molecules to understand the mode of action of active molecules.

\section{Materials and methods}

\section{Bacterial strains and culture conditions}

The bacterial strains used in this work were: $S$. epidermidis O-47 isolated from clinical septic arthritis and kindly provided by Professor Gotz; P. haloplanktis TAC125 (17) was collected in 1992 from seawater near the French Antarctic Station Dumont d'Urville $\left(60^{\circ} 40^{\prime} ; 40^{\circ} 01^{\prime} \mathrm{E}\right)$. Bacteria were grown in Brain Heart Infusion broth (BHI, Oxoid, UK) and synthetic medium GG (10 g/L D-Gluconic acid sodium, $10 \mathrm{~g} / \mathrm{L}$ glutammic acid, SCHATZ salt mixture). Biofilm formation was assessed in static condition while planktonic cultures were performed under vigorous agitation $(180 \mathrm{rpm})$. All strains were maintained at $-80^{\circ} \mathrm{C}$ in cryovials with $15 \%$ of glycerol.

\section{Biofilm formation of P. haloplanktis TACI 25}

Static biofilm assay. Quantification of in vitro biofilm production was based on the method described by Christensen with slight modifications. ${ }^{18}$ Briefly, the wells of a sterile 24 -well flatbottomed polystyrene plate were filled with $1 \mathrm{~mL}$ of BHI, and an suitable dilution of Antarctic bacterial culture in exponential growth phase (about $0.1 \mathrm{OD} 600 \mathrm{~nm}$ ) was added into each well. The sterile 24-well flat-bottomed polystyrene plates were incubated for different times $(24 \mathrm{~h}, 48 \mathrm{~h}, 72$ $\mathrm{h}, 96 \mathrm{~h}$, and $120 \mathrm{~h}$ ) at $4{ }^{\circ} \mathrm{C}$. After rinsing with PBS, adhered cells were stained with $0.1 \%$ crystal violet, rinsed twice with double-distilled water, and thoroughly dried. The dye bound to adherent cells was solubilized with $20 \%(\mathrm{v} / \mathrm{v})$ acetone and $80 \%$ $(\mathrm{v} / \mathrm{v})$ ethanol. The OD of each well was measured at $590 \mathrm{~nm}$. Each data point is composed of four independent samples.

\section{Preparation of $\mathrm{P}$. haloplanktis TACI 25 supernatants}

$\mathrm{SN}$ is the supernatant of a liquid culture of $P$. haloplanktis TAC125 grown without shaking in $\mathrm{BHI}$ at different temperatures $\left(4^{\circ} \mathrm{C}, 15^{\circ} \mathrm{C}, 20^{\circ} \mathrm{C}\right)$. The wells of a sterile 24-well flat-bottomed polystyrene plate were filled with $900 \mathrm{~mL}$ of appropriate medium (BHI or GG) and $100 \mathrm{~mL}$ of overnight $P$. 
haloplanktis TAC125 bacterial culture was added into each well. The plates were incubated at different temperatures as already reported for different times (24 h, 48 h, 72 h, 96 h, and 120 h). P. haloplanktis TAC125 biofilm formation was monitored each $24 \mathrm{~h}$. Supernatants were recovered and separated from cells by centrifugation at 13,000 rpm. Then, they were sterilized by filtration through membranes with a pore diameter of $0.22 \mu \mathrm{m}$, and stored at $4^{\circ} \mathrm{C}$ until use.

\section{Biofilm formation of staphylococci}

Static biofilm assay. Quantification of in vitro biofilm production was based on the method described by Christensen with slight modifications. ${ }^{18}$ Briefly, the wells of a sterile 48-well flat-bottomed polystyrene plate were filled with $400 \mu \mathrm{L}$ of BHI medium; $1 / 100$ dilution of overnight bacterial cultures was added to each well. The first row contained the untreated bacteria, while each of the remaining rows contained serial dilutions of supernatant (SN) starting from $1: 2$. The plates were incubated aerobically for $24 \mathrm{~h}$ at $37^{\circ} \mathrm{C}$. After rinsing with PBS, adhered cells were stained with $0.1 \%$ crystal violet, rinsed twice with double distilled water, and thoroughly dried. The dye bound to adherent cells was resolubilized with $20 \%(\mathrm{v} / \mathrm{v})$ acetone and $80 \%$ $(\mathrm{v} / \mathrm{v})$ ethanol. The OD of each well was measured at $590 \mathrm{~nm}$. Each data point was composed of four independent samples.

Dynamic biofilm assay. To continuously monitor biofilm development in dynamic condition, we utilized a BioFlux 2000 microfluidic system (Fluxion Biosciences Inc., San Francisco, CA, USA), which allows the acquisition of microscopic images over time using the experimental protocol reported by Papa and co-workers. ${ }^{19}$ Each flow channel connects to an input well (inlet) and an output well (outlet) on the plate. To grow biofilm in the BioFlux system, the channels were first primed. We filled the outlet with $100 \mu \mathrm{L}$ of sterile distilled water with flow at a share setting of $1 \mathrm{dyne} / \mathrm{cm}^{2}$ for $2 \mathrm{~min}$. Coating with $100 \mu \mathrm{L}$ of $10 \mu \mathrm{g} / \mathrm{mL}$ fibronectin was carried out for $2 \mathrm{~min}$ at $1 \mathrm{dyne} / \mathrm{cm}^{2}$. The fibronectin binding was performed for $30 \mathrm{~min}$ without flow. After priming, fibronectin was aspirated from the output wells and replaced with 100 $\mu \mathrm{L}$ of fresh overnight cultures diluted to an OD 600 of 0.8 . The channels were seeded by pumping from the output wells to the input wells at 2.0 dyne $/ \mathrm{cm}^{2}$ for $4 \mathrm{~s}$. Bacterial adhesion was performed for 30 min at $37^{\circ} \mathrm{C}$ without flow. A total of $2.0 \mathrm{~mL}$ of BHI was added to the input well and pumped at 1 dyne/ $\mathrm{cm}^{2}$ for $12 \mathrm{~h}$. We used two inlet wells; in the first well we added $\mathrm{F}$ fraction at a concentration of 1 $\mathrm{mg} / \mathrm{mL}$. In the second well we added only BHI. Bright-field images were taken at $40 \mathrm{X}$ magnification at 1-min intervals for a total of 720 time points.

$\mathrm{SN}$ treatment with $\mathrm{NalO4}$. For NaIO4 treatment, it was added at a final concentration of $20 \mathrm{mM}$ to sample for $12 \mathrm{~h}$ at $37^{\circ} \mathrm{C}$. As control, the same treatment was performed on BHI broth to exclude an anti-biofilm effect due to the NaIO4 itself. After treatment, samples were sterilized by filtration through membranes with a pore diameter of 0.22 $\mu \mathrm{m}$, and stored at $4^{\circ} \mathrm{C}$ until use.

Surface coating assay. A volume of $25 \mathrm{~mL}$ of $P$. haloplanktis cell-free supernatant, or $25 \mathrm{~mL}$ of saline and BHI as controls, were transferred to the center of a well of a 24-well tissue-culture-treated polystyrene microtiter plate. The plate was incubated at room temperature to allow complete evaporation of the liquid. The wells were then filled with $1 \mathrm{~mL}$ of broth containing a 1/100 dilution of $S$. epidermidis overnight bacterial cultures and incubated at $37^{\circ} \mathrm{C}$ in static condition. After $18 \mathrm{~h}$, the wells were rinsed with water and stained with $1 \mathrm{~mL}$ of $0.1 \%$ crystal violet. Stained biofilms were rinsed with water and dried, and the wells were photographed.

Anti-biofilm molecule purification. The first step was a dialysis against MilliQ water of $P$. haloplanktis TAC125 supernatant deriving sessile growth at $4^{\circ} \mathrm{C}$. In this procedure a semipermeable membrane with a cutoff of 3,500 Da was used. The dialysate water was subjected to a gel-filtration liquid chromatography on a Biogel P-2 column (Bio-Rad, molecular mass separation range 100-1800 Da, 60 $\times 0.75 \mathrm{~cm}$, flow rate $15 \mathrm{~mL} / \mathrm{h}$, eluent water, fraction volume $1 \mathrm{~mL}$ ).

The fraction resulting as active was further fractionated by high-performance liquid chromatography on a C18 column (Kinetex, Phenomenex, 150 $\times 4.6 \mathrm{~mm}$ ) eluting with the following program: $1 \%$ of $\mathrm{B}$ for $10 \mathrm{~min}(\mathrm{~A}: \mathrm{H} 2 \mathrm{O}, \mathrm{B}$ : $\mathrm{AcCN}), 1$ to $95 \%$ of $\mathrm{B}$ in $10 \mathrm{~min}, 95 \%$ of $B$ for $10 \mathrm{~min}$, flow rate $1 \mathrm{~mL} /$ $\min )$. 


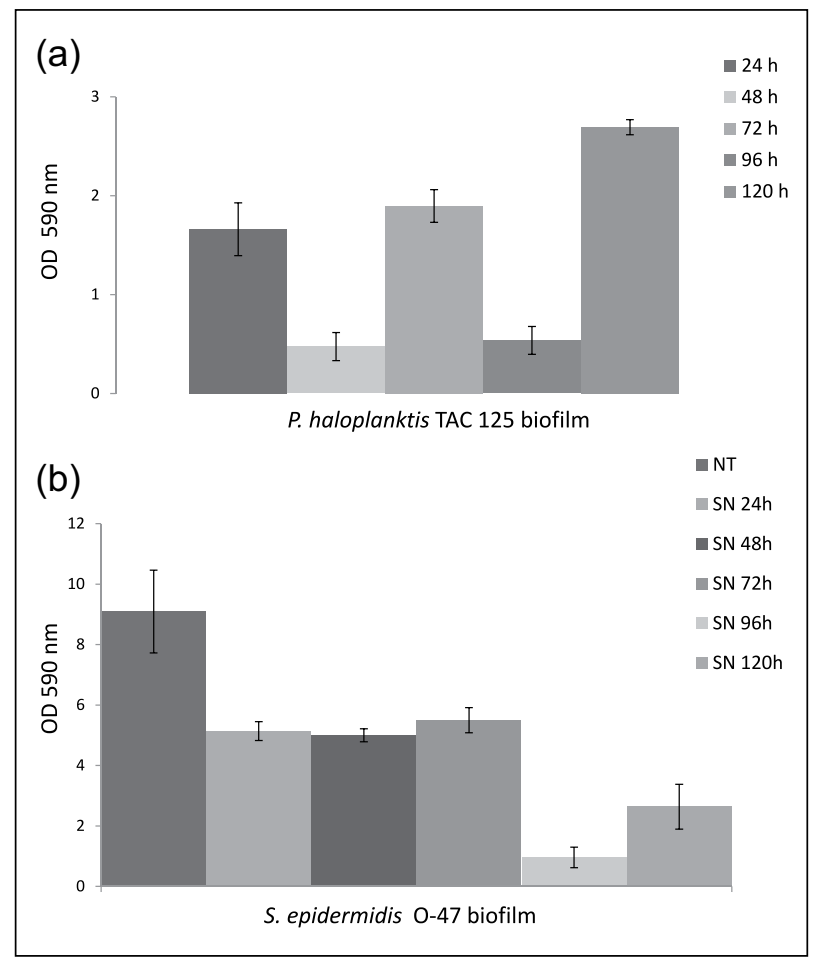

Figure I. Kinetic biofilm development in P. haloplanktis TACI 25 and production of anti-biofilm activity against $\mathrm{S}$. epidermidis O-47. (a) Evaluation of P. haloplanktis biofilm formation at different times. (b) Biofilm formation of S. epidermidis strain O-47 treated with $P$. haloplanktis TACI 25 supernatants deriving from sessile cultures obtained at different times.

\section{Results}

Optimization of P. haloplanktis TACI 25 antibiofilm molecule production conditions

Study of the effect of bacterial biofilm kinetics on P. haloplanktis TACI 25 anti-biofilm molecule production. Previously reported results demonstrated that the production of the $P$. haloplanktis TAC125 antibiofilm compounds was dependent on bacterial growth modality, ${ }^{15}$ indeed the production of antibiofilm compounds only occurs if Antarctic cells were grown in static condition. In order to assess whether the anti-biofilm was produced in all phases of biofilm development cycle, its formation over a 120 -h period in $\mathrm{BHI}$ at $4^{\circ} \mathrm{C}$ was evaluated (Figure 1a). As shown in Figure 1a, P. haloplanktis TAC125 biofilm kinetics is characterized by phases of biofilm development ( $24 \mathrm{~h}, 72 \mathrm{~h}, 120 \mathrm{~h}$ ) and phases of cells detachment (at 48 h, 96 h). Supernatants (SN) collected at different stages were tested to assess their effect on $S$. epidermidis strain O-47 biofilm formation (Figure 1B). In detail, supernatants of $P$. haloplanktis TAC125 cultures grown in sessile

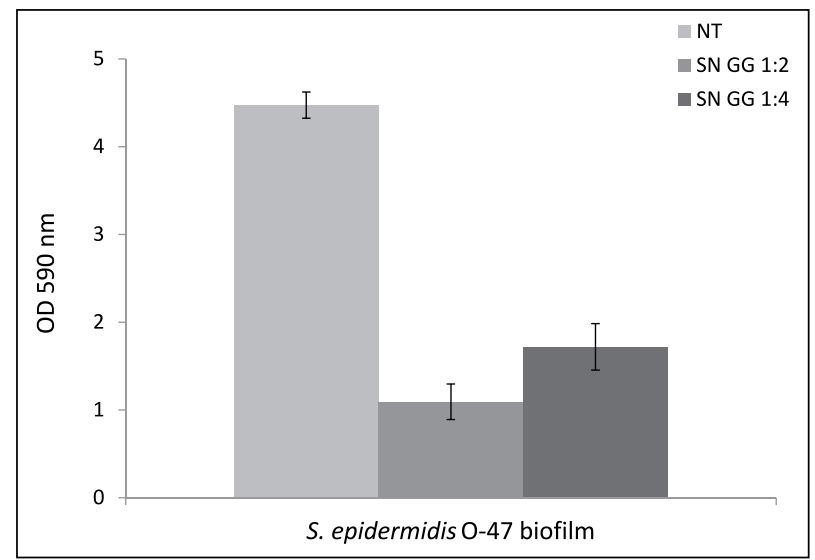

Figure 2. Biofilm formation of $S$. epidermidis $0-47$ in minimal medium GG in the presence and in the absence of scalar dilutions of $P$. haloplanktis supernatant. P. haloplanktis was grown in GG minimal medium in sessile condition.

condition at the different times (SN $24 \mathrm{~h}, \mathrm{SN} 48 \mathrm{~h}$, SN 72 h, SN 96 h, SN 120 h) were recovered and their effects on $S$. epidermidis strain O-47 biofilm were analyzed (Figure 1B). Reported data demonstrated that the anti-biofilm molecule is produced in all $P$. haloplanktis TAC125 biofilm stages although the best production is obtained at $96 \mathrm{~h}$ (Figure 1b); in fact, the supernatant collected at 96 $\mathrm{h}$ is able to decrease the biofilm formation of $S$. epidermidis strain O-47 about of $91 \%$. Study of the effect of different culture media on $P$. haloplanktis TAC125 anti-biofilm compound production to evaluate whether the culture medium composition has influence on bioactive compound production, $P$. haloplanktis TAC125 was grown in a synthetic medium (based on gluconate and glutamate (GG)) in sessile condition at $4^{\circ} \mathrm{C}$ for a $96-\mathrm{h}$ period. Then the effect of the obtained supernatant (SN-GG) on S. epidermidis strain O-47 biofilm was evaluated (Figure 2). In this experiment, $S$. epidermidis strain O-47 was grown in GG medium to avoid interference from the medium composition. As shown in Figure 2, the biofilm formation of $S$. epidermidis strain $\mathrm{O}-47$ in the GG medium was lower than the BHI culture medium (Figure 1b), furthermore the SN-GG inhibitory effect is evident and dosedependent. The treatment with SN-GG at a dilution of 1:2 induced a decrease in biofilm formation of $S$. epidermidis strain O-47 of approximately $85 \%$.

Study of the effect of different growth temperatures on P. haloplanktis TACI 25 anti-biofilm compound production. In order to assess whether growth temperature 


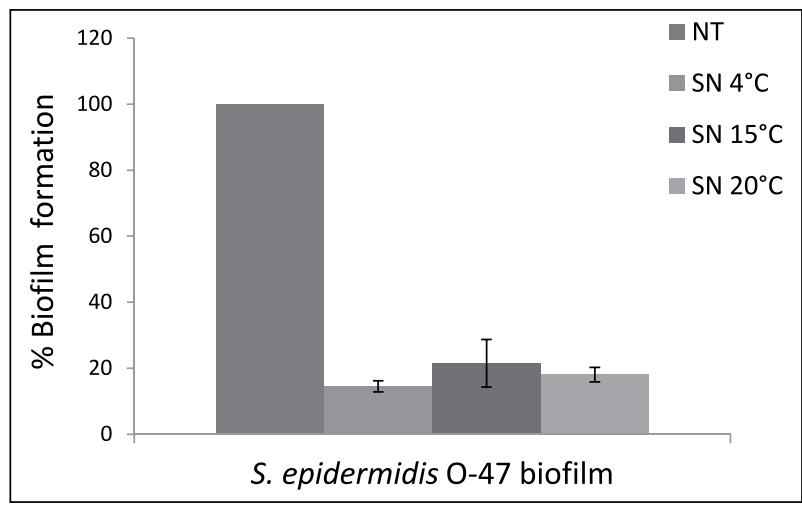

Figure 3. Biofilm formation of $S$. epidermidis $O-47$ in the presence and in the absence of $P$. haloplanktis supernatant deriving from sessile cultures performed at different temperatures. Data are reported as percentage of residual biofilm after the treatment.

has influence on anti-biofilm compound production, the Antarctic bacterium was grown in sessile condition at different temperatures $\left(4^{\circ} \mathrm{C}, 15^{\circ} \mathrm{C}\right.$, $20^{\circ} \mathrm{C}$ ). Corresponding supernatants were recovered for each condition after $96 \mathrm{~h}$ at $4^{\circ} \mathrm{C}, 96 \mathrm{~h}$ at $15^{\circ} \mathrm{C}$, and $48 \mathrm{~h}$ at $20^{\circ} \mathrm{C}$, and their effect on S. epidermidis strain O-47 biofilm was evaluated (Figure 3). As shown in Figure 3 the anti-biofilm compound was produced at all tested temperatures $\left(4-20^{\circ} \mathrm{C}\right)$, demonstrating that anti-biofilm production is not temperature-dependent.

\section{P. haloplanktis TACI 25 anti-biofilm molecule is not a polysaccharide}

Previous reported results suggested that $P$. haloplanktis TAC125 anti-biofilm activity could be due to a polysaccharide molecule. ${ }^{15}$ Many antibiofilm polysaccharides act as a surfactant molecule that modifies the physical characteristics of bacterial cells and abiotic surfaces. Therefore it was tested whether P. haloplanktis TAC125 supernatant could modify the surface properties of an abiotic substrate. To do this, evaporation coating was used to deposit the supernatant onto the surface of polystyrene wells, and then the ability of the coated surfaces to impair biofilm formation by $S$. epidermidis was tested. The supernatant deriving from sessile growth of $P$. haloplanktis at $4^{\circ} \mathrm{C}$ for $96 \mathrm{~h}$ was collected and used. When SN was applied to the polystyrene surfaces, the coated surfaces was not able to repel $S$. epidermidis biofilm formation in the area where the extract was deposited (data not shown), indicating that $\mathrm{SN}$ did not act as a surfactant.

To further investigate the anti-biofilm nature, we started a preliminary purification of bioactive molecules from 96-h culture supernatant. Usually, a polysaccharide molecule can be separated from culture medium using a dialysis membrane with a 3,500 Da cutoff: in these conditions the polysaccharide is retained inside the dialysis tube. P. haloplanktis TAC125 supernatant deriving from sessile growth at $4{ }^{\circ} \mathrm{C}$ was subjected to dialysis treatment and the sample retained inside the dialysis tube was subjected to size-exclusion chromatography (Sephacryl S-200). The retained sample and each chromatographic collected fraction were assayed in order to assess their anti-biofilm activity against $S$. epidermidis strain O-47 (data not shown). Results obtained demonstrated that none of the tested samples showed anti-biofilm activity (data not shown). Therefore, the anti-biofilm activity of dialysis permeate was evaluated and resulted to be active (data not shown). Consequently the bioactive molecules possessed a molecular mass lower than 3,500 Da, a molecular size not compatible with a polysaccharide.

It has been shown that NaIO4 is able to oxidize the carbons bearing vicinal hydroxyl groups and to cleave the $\mathrm{C}-\mathrm{C}$ bonds; this oxidizing activity was demonstrated also against polysaccharides. ${ }^{20}$ The permeate was treated with $\mathrm{NaIO} 4$, for the above tests, the anti-biofilm activities of treated and untreated permeated were compared. The result of this experiment showed that $\mathrm{NaIO} 4$ did not have effect on permeate anti-biofilm activity (data not shown). These data demonstrate that the reported anti-biofilm activity is not due to a polysaccharide molecule.

\section{Partial purification of $\mathrm{P}$. haloplanktis TACI 25 anti-biofilm compounds}

P. haloplanktis TAC125 supernatant deriving from sessile growth at $4^{\circ} \mathrm{C}$ was subject to dialysis treatment using a semipermeable membrane with a cutoff of $3500 \mathrm{Da}$. Both the retained and the permeate from the dialysis tube were tested for antibiofilm activity and only the second one was shown to be active. The presence of a complex mixture in the permeate was shown by $1 \mathrm{H} \mathrm{NMR}$ spectrum of the sample (data not shown). Consequently, a liquid chromatography on a 


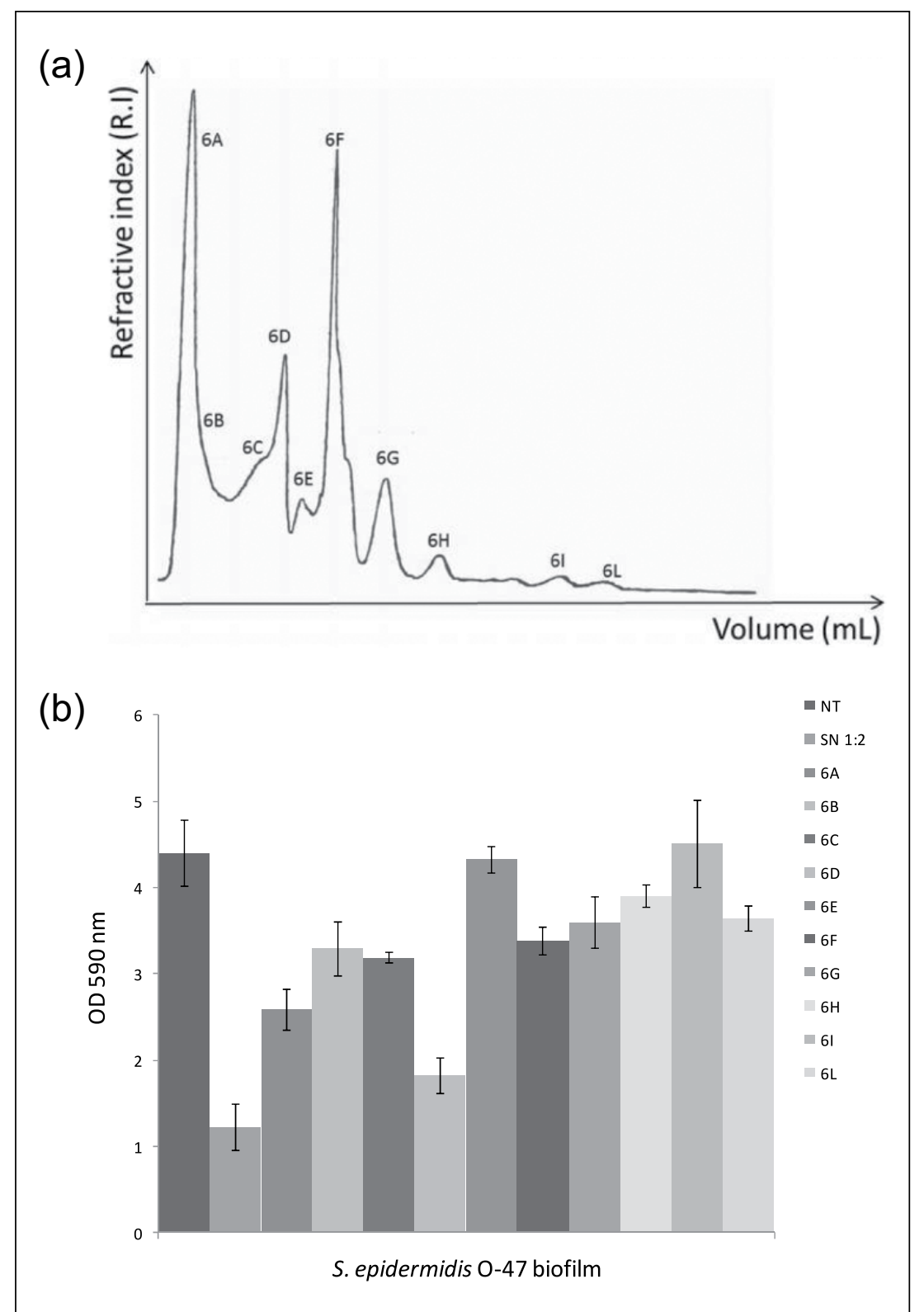

Figure 4. Purification of active compound from $P$. haloplanktis supernatant. (a) LC chromatographic profile of $P$. haloplanktis supernatant. (b) Biofilm formation by S. epidermidis $0-47$ in the presence and in the absence of exclusion chromatography fractions.

Biogel P-2 column was performed to fractionate the dialysate sample (Figure $4 \mathrm{a}$ ) and the biological activity of each fraction was evaluated (Figure $4 b)$. The obtained results indicated for the fraction $6 \mathrm{D}$ the best anti-biofilm activity, but once again $1 \mathrm{H}$ NMR spectrum revealed the presence of a complex mixture (data not shown). Reversephase HPLC on a C18 column was used to further purify $6 \mathrm{D}$ fraction and, the sub-fractions derived from this procedure were tested to evaluate the anti-biofilm activity on $S$. epidermidis $\mathrm{O}-47$ biofilm. The results (Figure 5) clearly showed that the treatment with $\mathrm{F}$ fraction induces a decrease of the biofilm formation of $S$. epidermidis strain O-47 about of $90 \%$.

1H NMR spectrum suggested that the fraction $\mathrm{F}$ still is a mixture. However, proton NMR spectra obtained during the purification steps gave further confirmation that the molecule of interest in not a carbohydrate. 


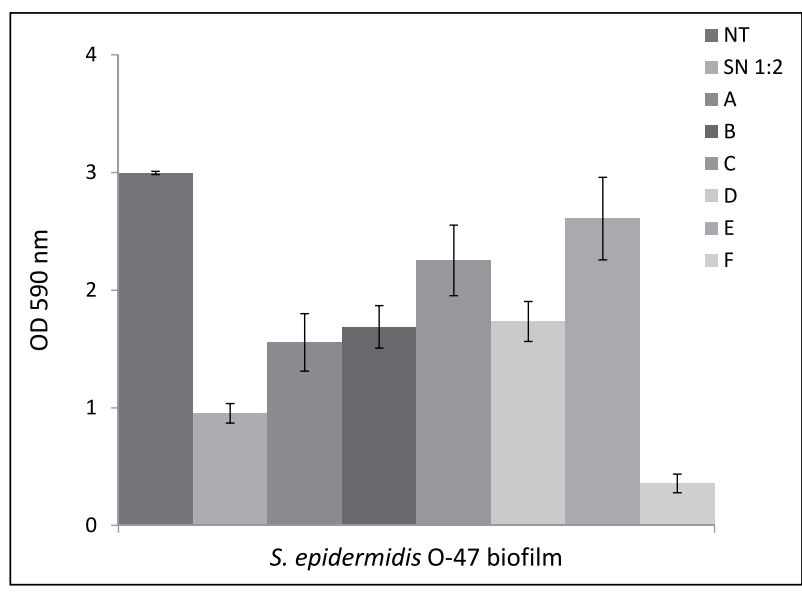

Figure 5. Biofilm formation by S. epidermidis $\mathrm{O}-47$ in the presence and in the absence of HPLC chromatography fractions.

Effect of partially purified active compound(s) on S. epidermidis 0-47 dynamic biofilm formation

Effect of F fraction treatment on $S$. epidermidis O-47 biofilm formation was also evaluated on BioFlux system. ${ }^{21}$ The BioFlux system is a microfluidic device that precisely controls the flow of growth medium between two interconnected wells of a microtiter plate. By positioning the channel connecting the two wells over a window accessible for viewing by microscopy, biofilm growth can be monitored in a time-course assay in which images are collected at 1-min intervals. We collected 720 frames for each experiment, assembled in a timelapse video. Selected images reported in Figure 6 show the biofilm development of $S$. epidermidis O-47 at different times, in the absence and in the presence of $\mathrm{F}$ fraction (bottom and top lanes of each panel, respectively).

Bacteria were seeded in both channels visible in each frame. After 30 min the flow was applied. In the top channel $\mathrm{F}$ fraction was added to the medium, while the bottom one contained only medium. Data obtained show an initial rapid growth of the bacteria, resulting in a confluent 'lawn' of cells that was followed by a period of detachment. $F$ fraction clearly impaired the biofilm formation confirming results obtained in static system.

\section{Discussion}

In a previous paper ${ }^{15}$ we demonstrated that the cellfree supernatant of Antarctic bacterium P. haloplanktis TAC125 was effective on $S$. epidermidis biofilm even on the mature form. Moreover, the $P$. haloplanktis TAC125 supernatant resulted to have no antibacterial activity against free-living bacteria, and results to be species-specific. ${ }^{15}$ Interestingly $P$. haloplanktis TAC125 produces this activity only when it is grown in sessile condition. This latter result could be explained in consideration that specific environmental conditions prevailing within biofilms may induce profound genetic and metabolic rewiring of the biofilm-dwelling bacteria, ${ }^{22}$ which could lead to production of biofilm-specific metabolites. These peculiar features could be compatible with the action of different anti-biofilm molecules, such as polysaccharides, ${ }^{9}$ biosurfactants, ${ }^{22}$ quorum sensing inhibitors, ${ }^{23,24}$ or signaling molecule that modulates the gene expression of recipient bacteria. ${ }^{25}$

In this work we aimed to assess which kind of anti-biofilm molecules are produced by the Antarctic bacterium. To collect information on anti-biofilm compound characteristics we explored the dependence of bioactive molecule production to growth conditions. Reported results demonstrated that the anti-biofilm compound is produced during all phases of $P$. haloplanktis TAC125 biofilm development with the best production of the molecules corresponding to biofilm detachment steps, and that the production is independent from the specific carbon and nitrogen source and is not temperature-dependent.

Previously reported data suggested that $P$. haloplanktis TAC 125 anti-biofilm compound is not a surfactant molecule because its action on mature biofilm is not immediate but requires a prolonged time. ${ }^{15}$ In this paper we confirmed this hypothesis by surface coating assay, the reported result demonstrated that $P$. haloplanktis TAC125 supernatant did not modify the surface properties of abiotic substrate.

Preliminary physico-chemical characterization of supernatant of $P$. haloplanktis TAC125 suggested the hypothesis that the anti-biofilm molecule has a polysaccharidic nature, ${ }^{15}$ in which case the data presented in the present paper disavow this hypothesis.

The setting up of a purification protocol allowed us to obtain a fraction ( $F$ fraction), that does not contain polysaccharides, enriched in anti-biofilm compound. The proposed purification protocol has to be optimized since its purification yield is very low, but it is interesting to note that $\mathrm{F}$ fraction, 


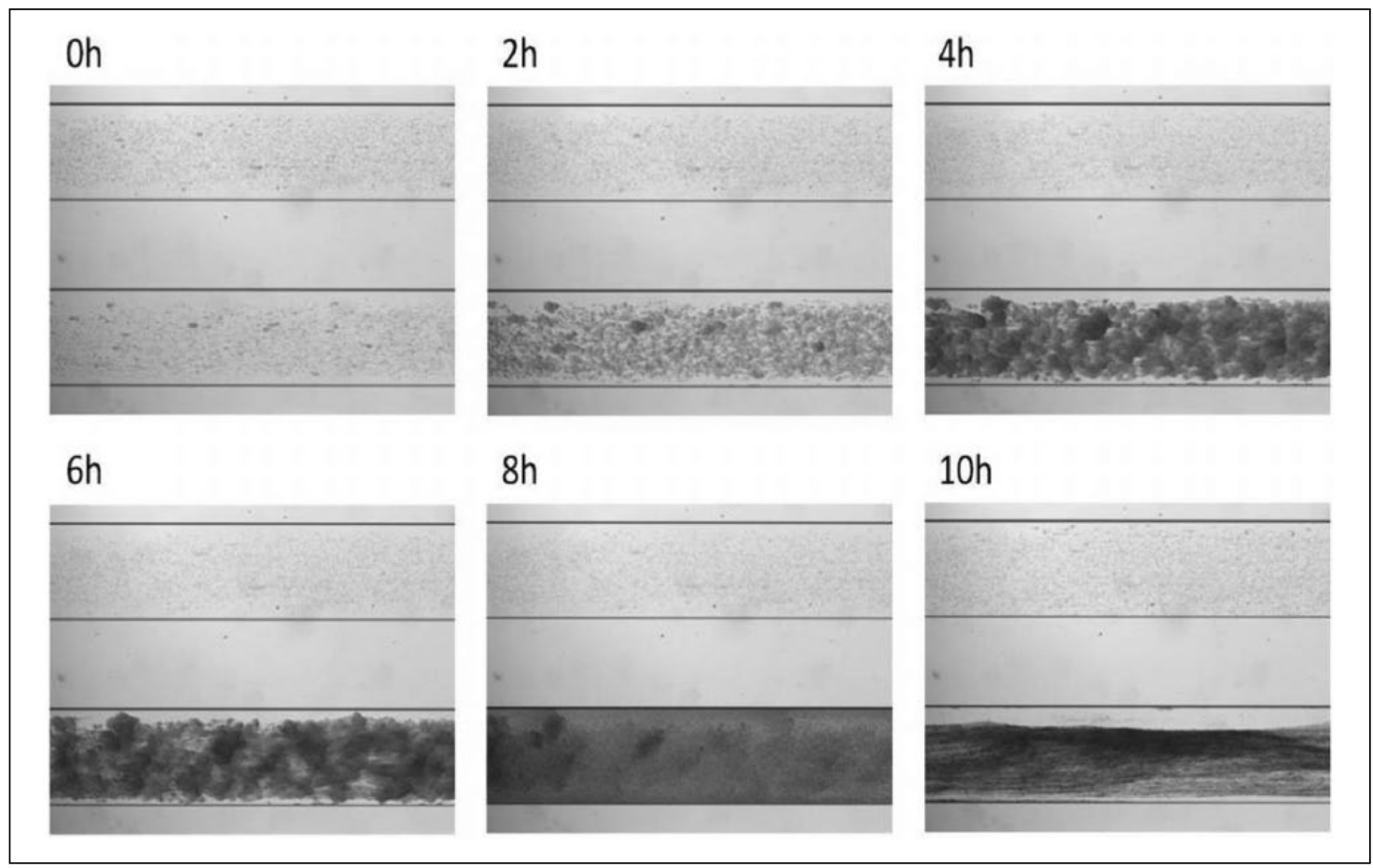

Figure 6. Biofilm formation of S. epidermidis $\mathrm{O}-47$ in a Bioflux system. Each image contains two channels: top channel was SNtreated sample and bottom channel was the control one. Bright-field microscopic images were collected at I-min intervals. The images presented were taken from the complete set of 720 images (see supplementary video bioflux for a video compilation of these images) taken at $40 \times$ magnification.

which is still a mixture of several compounds, results as being active at a concentration lower than $1 \mathrm{mg} / \mathrm{mL}$, suggesting that anti-biofilm molecule resulted as being endowed with a strong biological activity. Moreover, the molecules contained in the $\mathrm{F}$ fraction have a molecular weight smaller than 3,500 Da and display a hydrophobic character as indicated by $\mathrm{F}$ fraction elution time in reversephase chromatography. Therefore, reported results demonstrate that the P. haloplanktis TAC125 antibiofilm activity is due to a small hydrophobic molecule, able to affect $S$. epidermidis biofilm formation and stability.

The $P$. haloplanktis TAC125 anti-biofilm molecule is active against several $S$. epidermidis strains, among others it is effective on the clinical isolate $\mathrm{O}-47$, which is a naturally occurring agr mutant, ${ }^{26}$ but it is inactive on $S$. epidermidis XX-17 ica mutant $^{27}$ (data not shown). The ica operon encodes enzymes responsible for production of polysaccharide intercellular adhesin (PIA or PNAG), which is the main component of polysaccharide matrix of staphylococcus biofilm. ${ }^{28}$ These data could indicate in the ica genes the possible target of antibiofilm molecules. ica genes regulation in $S$. epidermidis is under the control of LuxS quorum sensing system. ${ }^{28}$ It was reported that deletion of lux $\mathrm{S}$ gene in S. epidermidis enhances biofilm formation, this effect being due to an increase over four-fold of ica expression and resulting increase of PIA synthesis. ${ }^{29}$

Therefore, the LuxS communication system could be involved in bacterial intra-species communication between $S$. epidermidis and P. haloplanktis TAC125. The Antarctic bacterium genome analysis revealed that it is devoid of the $\operatorname{luxS}$ gene, ${ }^{17}$ therefore the anti-biofilm effects of $P$. haloplanktis exoproducts could be due to a novel molecule, or to the synergistic actions of different molecules, that could work as an AI-2 agonist or as a ligand able to target the AI-2 receptor inducing an AI-2 signaling response. Only the full structural characterization of the active molecule will address the detailed mode of action of anti-biofilm molecules. However, regardless of the chemical nature of the anti-biofilm molecule, its efficacy was confirmed on $S$. epidermidis biofilm formation in dynamic condition using the BioFlux system.

BioFlux is an accurate representation of environmental or physiological conditions by precisely 
controlling shear flow and can bridge the gap between in vitro and in vivo assays. Under these conditions, the bacteria progress through a series of developmental steps, ultimately forming a multicellular structure containing differentiated cell populations. The observation of the biofilm at various time-points throughout this process provides a glimpse of the temporal changes that occur.

Flow biofilm is closer to natural biofilms and can differ from static biofilms, evidently due to hydrodynamic influences on cell signaling. The reported results demonstrate that the $P$. haloplanktis TAC 125 anti-biofilm is effective against $S$. epidermidis biofilm formation in dynamic condition; this conclusion is very promising with regard to its use in in vivo systems. Therefore the use of $P$. haloplanktis TAC125 anti-biofilm molecules during persistent infection sustained by staphylococci in combination therapy with antibiotics could be proposed.

\section{Declaration of conflicting interests}

The author(s) declared no potential conflicts of interest with respect to the research, authorship, and/or publication of this article.

\section{Funding}

This work was supported by Programma Nazionale di Ricerche in Antartide 2013/B1.04 Tutino.

\section{References}

1. Dohar JE, Roland P, Wall GM et al. (2009) Differences in bacteriologic treatment failures in acute otitis externa between ciprofloxacin/dexamethasone and neomycin/polymyxin B/hydrocortisone: Results of a combined analysis. Current Medical Research and Opinion 25: 287-291.

2. Rogers KL, Fey PD and Rupp ME (2009) Coagulasenegative staphylococcal infections. Infectious Disease Clinics of North America 23: 73-98.

3. Costerton JW, Stewart PS and Greenberg EP (1999) Bacterial biofilms: A common cause of persistent infections. Science 284: 1318-1322.

4. Otto M (2012) Molecular basis of Staphylococcus epidermidis infections. Seminars in Immunopathology 34: 201-214.

5. Ni N, Li M, Wang J et al. (2009) Inhibitors and antagonists of bacterial quorum sensing. Medicinal Research Reviews 29: 65-124.

6. Kiran GS, Sabarathnam B and Selvin J (2010) Biofilm disruption potential of a glycolipid biosurfactant from marine Brevibacterium casei. FEMS Immunology and Medical Microbiology 59: 432-438.
7. Kaplan JB (2010) Biofilm dispersal: Mechanisms, clinical implications, and potential therapeutic uses. Journal of Dental Research 89: 205-218.

8. Qin Z, Yang L, Qu D et al. (2009) Pseudomonas aeruginosa extracellular products inhibit staphylococcal growth, and disrupt established biofilms produced by Staphylococcus epidermidis. Microbiology 155: 2148-2156.

9. Valle J, Da Re S, Henry N et al. (2006) Broadspectrum biofilm inhibition by a secreted bacterial polysaccharide. Proceedings of the National Academies of Science of the United States of America 103: 12558-12563.

10. Brook I (1999) Bacterial interference. Critical Reviews in Microbiology 25: 155-172.

11. Wang CZ, Li M, Dong D et al. (2007) Role of ClpP in biofilm formation and virulence of Staphylococcus epidermidis. Microbes and Infection 9: 1376-1383.

12. Wang CZ, Fan JJ, Niu C et al. (2010) Role of spx in biofilm formation of Staphylococcus epidermidis. FEMS Immunology and Medical Microbiology 59: 152-160.

13. Klein GL, Soum-Soutera E, Guede Z et al. (2011) The anti-biofilm activity secreted by a marine Pseudoalteromonas strain. Biofouling 27: 931-940.

14. Jayatilake GS, Thornton MP, Leonard AC et al. (1996) Metabolites from an Antarctic sponge-associated bacterium, Pseudomonas aeruginosa. Journal of Natural Products 59: 293-296.

15. Papa R, Parrilli E, Sannino F et al. (2013) Antibiofilm activity of the Antarctic marine bacterium Pseudoalteromonas haloplanktis TAC125. Research in Microbiology 164: 450-456.

16. Papaleo MC, Romoli R, Bartolucci G, et al. (2013) Bioactive volatile organic compounds from Antarctic (sponges) bacteria. New Biotechnology 30: 824-38.

17. Medigue C, Krin E, Pascal G, et al. (2005) Coping with cold: The genome of the versatile marine Antarctica bacterium Pseudoalteromonas haloplanktis TAC125. Genome Research 15: 1325-1335.

18. Artini M, Romano C, Manzoli L et al. (2011) Staphylococcal IgM enzyme-linked immunosorbent assay for diagnosis of periprosthetic joint infections. Journal of Clinical Microbiology 49: 423-425.

19. Papa R, Artini M, Cellini A et al. (2013) A new antiinfective strategy to reduce the spreading of antibiotic resistance by the action on adhesion-mediated virulence factors in Staphylococcus aureus. Microbial Pathogenesis 63: 44-53.

20. Bendaoud M, Vinogradov E, Balashova NV et al. (2011) Broad-spectrum biofilm inhibition by Kingella kingae exopolysaccharide. Journal of Bacteriology 193: 3879-3886.

21. Benoit MR, Conant CG, Ionescu-Zanetti $C$ et al. (2010) New device for high-throughput viability screening of 
flow biofilms. Applied and Environmental Microbiology 76: 4136-4142.

22. Beloin C, Michaelis K, Lindner K et al. (2006) The transcriptional antiterminator $\mathrm{RfaH}$ represses biofilm formation in Escherichia coli. Journal of Bacteriology 188: 1316-1331.

23. Das P, Mukherjee S and Sen R (2009) Antiadhesive action of a marine microbial surfactant. Colloids and Surfaces B: Biointerfaces 71: 183-186.

24. Rasmussen TB and Givskov M (2006) Quorum-sensing inhibitors as anti-pathogenic drugs. International Journal of Medical Microbiology 296: 149-161.

25. Estrela AB, Heck MG and Abraham WR (2009) Novel approaches to control biofilm infections. Current Medicinal Chemistry 16: 1512-1530.

26. Kim HS, Kim SM, Lee HJ et al. (2009) Expression of the cpdA gene, encoding a 3',5' -cyclic AMP (cAMP) phosphodiesterase, is positively regulated by the cAMP-cAMP receptor protein complex. Journal of Bacteriology 191: 922-930.

27. Vuong C, Gerke C, Somerville GA et al. (2003) Quorum-sensing control of biofilm factors in Staphylococcus epidermidis. Journal of Infectious Diseases 188: 706-718.
28. Xu L, Li HL, Vuong C et al. (2006) Role of the luxS quorum-sensing system in biofilm formation and virulence of Staphylococcus epidermidis. Infection and Immunity 74: 488-496.

29. Cue D, Lei MG and Lee CY (2012) Genetic regulation of the intercellular adhesion locus in staphylococci. Frontiers in Cellular and Infection Microbiology 2: 38.

30. Doherty N, Holden MT, Qazi SN et al. (2006) Functional analysis of luxS in Staphylococcus aureus reveals a role in metabolism but not quorum sensing. Journal of Bacteriology 188: 2885-2897.

31. Cluzel ME, Zanella-Cleon I, Cozzone AJ et al. (2010) The Staphylococcus aureus autoinducer-2 synthase LuxS is regulated by Ser/Thr phosphorylation. Journal of Bacteriology 192: 6295-6301.

32. Nichols JD, Johnson MR, Chou CJ et al. (2009) Temperature, not LuxS, mediates AI-2 formation in hydrothermal habitats. FEMS Microbiology Ecology 68: $173-181$.

33. Tavender TJ, Halliday NM, Hardie KR et al. (2008) LuxS-independent formation of AI-2 from ribulose5-phosphate. BMC Microbiology 8: 98. 